This item was submitted to Loughborough's Research Repository by the author.

Items in Figshare are protected by copyright, with all rights reserved, unless otherwise indicated.

\title{
Two-dimensional, phase modulated lattice sums with application to the
} Helmholtz Green's function

PLEASE CITE THE PUBLISHED VERSION

http://dx.doi.org/10.1063/1.4905732

PUBLISHER

(c) AIP Publishing LLC

VERSION

VoR (Version of Record)

LICENCE

CC BY-NC-ND 4.0

REPOSITORY RECORD

Linton, C.M.. 2019. "Two-dimensional, Phase Modulated Lattice Sums with Application to the Helmholtz Green's Function". figshare. https://hdl.handle.net/2134/17057. 


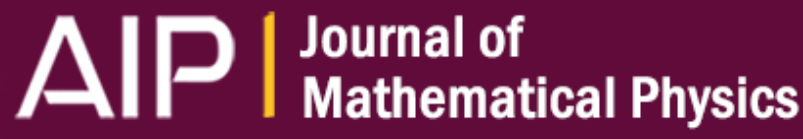

Two-dimensional, phase modulated lattice sums with application to the Helmholtz Green's function

C. M. Linton

Citation: Journal of Mathematical Physics 56, 013505 (2015); doi: 10.1063/1.4905732

View online: http://dx.doi.org/10.1063/1.4905732

View Table of Contents: http://scitation.aip.org/content/aip/journal/jmp/56/1 ?ver=pdfcov

Published by the AIP Publishing

\section{Articles you may be interested in}

Localized modes in mini-gaps opened by periodically modulated intersite coupling in two-

dimensional nonlinear lattices

Chaos 24, 023124 (2014); 10.1063/1.4881678

On the derivation of the Green's function for the Helmholtz equation using generalized functions Am. J. Phys. 78, 181 (2010); 10.1119/1.3253655

Closed form of the generalized Green's function for the Helmholtz operator on the two-dimensional unit sphere

J. Math. Phys. 47, 063506 (2006); 10.1063/1.2203430

Evaluation of phase-modulated lattice sums

J. Math. Phys. 45, 3584 (2004); 10.1063/1.1777403

Application of the lattice Green's function for calculating the resistance of an infinite network of resistors

Am. J. Phys. 68, 896 (2000); 10.1119/1.1285881

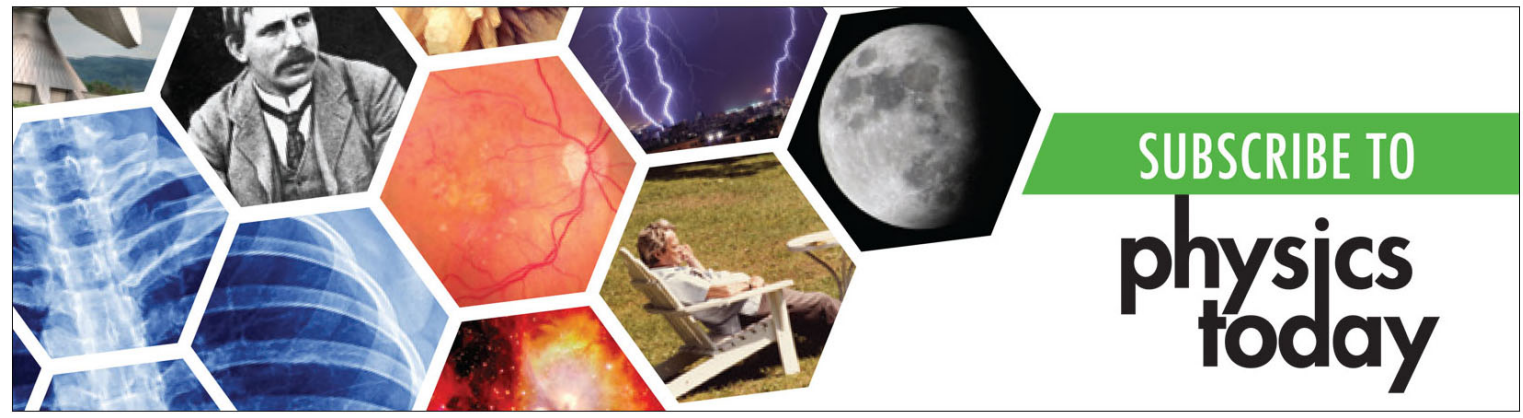




\title{
Two-dimensional, phase modulated lattice sums with application to the Helmholtz Green's function
}

\author{
C. M. Linton a) \\ Department of Mathematical Sciences, Loughborough University, Leicestershire LE11 3TU, \\ United Kingdom
}

(Received 3 October 2014; accepted 29 December 2014; published online 20 January 2015)

\begin{abstract}
A class of two-dimensional phase modulated lattice sums in which the denominator is an indefinite quadratic polynomial $Q$ is expressed in terms of a single, exponentially convergent series of elementary functions. This expression provides an extremely efficient method for the computation of the quasi-periodic Green's function for the Helmholtz equation that arises in a number of physical contexts when studying wave propagation through a doubly periodic medium. For a class of sums in which $Q$ is positive definite, our new result can be used to generate representations in terms of $\theta$-functions which are significant generalisations of known results. (C) 2015 AIP Publishing LLC. [http://dx.doi.org/10.1063/1.4905732]
\end{abstract}

\section{INTRODUCTION}

The study of two-dimensional phase modulated lattice sums of the form

$$
\sum_{m=-\infty}^{\infty} \sum_{n=-\infty}^{\infty} Q_{m n}^{-1} \mathrm{e}^{2 \pi \mathrm{i}(m \xi+n \eta)},
$$

where $Q_{m n}$ is a quadratic polynomial in $m$ and $n$ was initiated by Glasser (1974). Glasser showed how, when $Q_{m n}$ is of the form $m^{2}+\mu^{2} n^{2}$, this sum (with the $m=n=0$ term omitted) can be evaluated explicitly in terms of $\theta$-functions. Such sums arise when considering the Green's function for the Laplacian on a rectangular lattice; see, for example, Borwein et al. (2013, Sec. 3.3). Actually, as Glasser noted, his method applies to the more general class of sums in which $Q_{m n}=a m^{2}+2 b m n+c n^{2}$, provided $Q_{m n}$ is always positive ( $m=n=0$ excepted of course). This more general expression, which allows for lattices whose basis vectors are not orthogonal, was derived in Stremler (2004) and applied to a problem in vortex dynamics.

Glasser's method is briefly described as follows. First, the sum is generalised by raising $Q_{m n}$ to the power $s$. Provided the real part of $s$ is sufficiently large, this produces an absolutely convergent series which can be manipulated more easily. Next, $Q_{m n}^{-s}$ is written as the Mellin transform of an exponential function and the sum over one of the indices manipulated, resulting in an integral transform that can be evaluated in terms of modified Bessel functions. This then yields an exponentially convergent series (still a double sum). For the special case in which $s=1$, there is considerable simplification and one of the summations is simply a geometric progression which can be evaluated explicitly. Glasser showed how this could then be used to derive a representation in terms of $\theta$-functions. As well as providing a method for evaluating some two-dimensional, phase modulated lattice sums, Glasser's approach also shows how conditionally convergent sums of this type can usefully be considered as the analytic continuation of a more general complex function.

There are a number of extensions to the sums treated by Glasser, which are introduced in this paper. First, we generalise $Q_{m n}$ to include linear and constant terms. One of the consequences of this is that a general representation in terms of $\theta$-functions appears no longer to be possible. More fundamental is the extension to cases which include terms where $Q_{m n}<0$, for which there appears

\footnotetext{
a)Electronic mail: C.M.Linton@lboro.ac.uk
} 
to be very little previous work. The specific case considered here is driven by the application to the evaluation of the quasi-periodic Helmholtz Green's function, $G$, for a general two-dimensional lattice. The spectral representation for $G$ is a phase modulated sum in which $Q_{m n}$ is negative for a finite number of pairs $m, n$. Using results obtained via contour integration in Sec. II, we are able to derive, in Sec. III, an expression for a general sum of this type containing a single, exponentially convergent sum of elementary functions. We also show how Glasser's method can be modified so that sums of this type can still be considered as the analytic continuation of a more general complex function.

Many alternative expressions for this Green's function have been derived in an attempt to produce a form that leads to an efficient computational algorithm. Most notable is the use of Ewald summation (Ham and Segall (1961); Jordan et al. (1986); and Moroz (2006)) which produces a doubly infinite sum that is exponentially convergent, though for the case of a rectangular lattice, a method which leads to an exponentially convergent integral representation has also been developed by Dienstfrey et al. (2001); see also Linton (2010, Sec. 2.2.2). The approach described in Sec. IV would appear to provide the basis for an extremely effective computational scheme for the evaluation of $G$.

Finally, for a class of sums in which $Q_{m n}$ is positive definite, we can use the series derived in Sec. III to generate new representations for phase modulated lattice sums in terms of $\theta$-functions which are generalisations of known results. This is the subject of Sec. V.

Throughout this article, we use the convention that sums are over all integers unless explicitly stated otherwise. Hence, for example, $\sum_{m, n}$ is shorthand for $\sum_{m=-\infty}^{\infty} \sum_{n=-\infty}^{\infty}$. A dash on a summation indicates that any terms where the denominator vanishes are to be omitted.

\section{A CONTOUR INTEGRAL}

The results presented in this article all ultimately stem from the evaluation of a simple contour integral, which we describe first. Consider the function

$$
g(z)=\frac{\mathrm{e}^{\pi \mathrm{i}(2 \xi-1) z}}{\left(z-\zeta_{1}\right)\left(z-\zeta_{2}\right) \sin \pi z},
$$

where $0 \leq \xi \leq 1$, and $\zeta_{1}$ and $\zeta_{2}$ are arbitrary complex numbers. Consideration of the behaviour of this function around the large circle $|z|=N+1 / 2$ shows that

$$
\lim _{N \rightarrow \infty} \oint_{|z|=N+1 / 2} g(z) \mathrm{d} z=0 .
$$

If $\zeta_{1}$ and $\zeta_{2}$ are distinct and neither is an integer, then evaluation of the residues at $z=\zeta_{1}, z=\zeta_{2}$, and all integer values of $z$ shows that

$$
\sum_{m} \frac{\mathrm{e}^{2 \pi \mathrm{i} m \xi}}{\left(m-\zeta_{1}\right)\left(m-\zeta_{2}\right)}=\frac{\pi}{\zeta_{2}-\zeta_{1}}\left(\frac{\mathrm{e}^{\pi \mathrm{i}(2 \xi-1) \zeta_{1}}}{\sin \pi \zeta_{1}}-\frac{\mathrm{e}^{\pi \mathrm{i}(2 \xi-1) \zeta_{2}}}{\sin \pi \zeta_{2}}\right) .
$$

If $\zeta_{1}=\zeta_{2}=\zeta(\notin \mathbb{Z})$, there is a double pole and we obtain, either directly or by taking the limit as $\zeta_{2} \rightarrow \zeta_{1}$ in (3), the result

$$
\sum_{m} \frac{\mathrm{e}^{2 \pi \mathrm{i} m \xi}}{(m-\zeta)^{2}}=\frac{\pi^{2} \mathrm{e}^{2 \pi \mathrm{i} \zeta \xi}}{\sin ^{2} \pi \zeta}\left(1-\xi+\xi \mathrm{e}^{-2 \pi \mathrm{i} \zeta}\right) .
$$

If $\zeta_{1}=k$ is an integer but $\zeta_{2}$ is not, there is a double pole at $z=k$, simple poles at all other integers and at $z=\zeta_{2}$. This leads to

$$
\sum_{m}^{\prime} \frac{\mathrm{e}^{2 \pi \mathrm{i} m \xi}}{(m-k)\left(m-\zeta_{2}\right)}=\frac{\pi \mathrm{e}^{\pi \mathrm{i}(2 \xi-1) \zeta_{2}}}{\left(k-\zeta_{2}\right) \sin \pi \zeta_{2}}+\frac{\mathrm{e}^{2 \pi \mathrm{i} k \xi}}{\left(k-\zeta_{2}\right)^{2}}-\pi \mathrm{i}(2 \xi-1) \frac{\mathrm{e}^{2 \pi \mathrm{i} k \xi}}{k-\zeta_{2}} .
$$


If $\zeta_{1}=k_{1}$ and $\zeta_{2}=k_{2}$ are different integers then there are double poles at $z=k_{i}, i=1,2$ and simple poles at all other integers. In this case,

$$
\sum_{m}^{\prime} \frac{\mathrm{e}^{2 \pi \mathrm{i} m \xi}}{\left(m-k_{1}\right)\left(m-k_{2}\right)}=\frac{\mathrm{e}^{2 \pi \mathrm{i} k_{1} \xi}+\mathrm{e}^{2 \pi \mathrm{i} k_{2} \xi}}{\left(k_{1}-k_{2}\right)^{2}}+\pi \mathrm{i}(2 \xi-1) \frac{\mathrm{e}^{2 \pi \mathrm{i} k_{2} \xi}-\mathrm{e}^{2 \pi \mathrm{i} k_{1} \xi}}{k_{1}-k_{2}} .
$$

If $k_{1}=-k_{2}=k \neq 0$, this simplifies to

$$
\sum_{m}^{\prime} \frac{\mathrm{e}^{2 \pi \mathrm{i} m \xi}}{m^{2}-k^{2}}=\frac{\cos 2 \pi k \xi}{2 k^{2}}+\pi(2 \xi-1) \frac{\sin 2 \pi k \xi}{k} .
$$

Finally, if $\zeta_{1}=\zeta_{2}=k \in \mathbb{Z}$, there is a pole of order 3 at $z=k$ and we obtain

$$
\sum_{m}^{\prime} \frac{\mathrm{e}^{2 \pi \mathrm{i} m \xi}}{(m-k)^{2}}=\frac{\pi^{2}}{3} \mathrm{e}^{2 \pi \mathrm{i} k \xi}\left(1-6 \xi+6 \xi^{2}\right) .
$$

It is worth emphasising that all of the above results rely on the fact that $\xi \in[0,1]$. However, the extension to other real values of $\xi$ is trivial since the sums are all periodic in $\xi$ with period 1 .

\section{PHASE MODULATED LATTICE SUMS INVOLVING INDEFINITE QUADRATIC POLYNOMIALS}

We are concerned here with the lattice sum

$$
\mathcal{S}=\sum_{m, n} Q_{m n}^{-1} \mathrm{e}^{2 \pi \mathrm{i}(m \xi+n \eta)},
$$

where

$$
Q_{m n}=a m^{2}+2 b m n+c n^{2}+d m+e n+f, \quad a c-b^{2}>0 .
$$

The parameters $\xi, \eta, a, b, c, d, e, f$ are all real and we assume for convenience that $a>0$. The condition $a c-b^{2}>0$ ensures that for sufficiently large $|m|$ and $|n|, Q_{m n}>0$ or, in other words, that the number of pairs $(m, n)$ for which $Q_{m n}$ is negative is finite. For the time being, we assume that $Q_{m n}$ is never zero though examples where this condition is relaxed will also be considered. Without loss of generality, we can assume that both $\xi$ and $\eta$ lie in $[0,1)$ but they cannot both be zero as the resulting sum does not converge.

The sum $\mathcal{S}$ is then conditionally convergent, but it is known that if the sum is interpreted as the limit over rectangular lattices of increasing size, then the result will be independent of the nature of those rectangles. In fact, it is shown in Borwein et al. (1998) that sums of the form (9) converge to the same value when considered as the limit of expanding areas of any reasonable shape, the definition of reasonable certainly including rectangles (in the paper referred to above, $Q_{m n}$ is assumed to be positive definite but the results clearly extend to the case (10)). As a result, it is valid to perform the sum over $m$ first and then do the sum over $n$.

If we write

$$
Q_{m n}=a\left(m^{2}+\beta_{n} m+\gamma_{n}\right)
$$

then

$$
\beta_{n}=\frac{1}{a}(2 b n+d)
$$

and $\beta_{n}^{2}-4 \gamma_{n}=-4 \delta_{n}^{2}$, where

$$
a^{2} \delta_{n}^{2}=\left(a c-b^{2}\right) n^{2}+(a e-b d) n+a f-d^{2} / 4 .
$$

The roots of $z^{2}+\beta_{n} z+\gamma_{n}=0$ are, therefore, $z=\mu_{n}^{ \pm}$, where

$$
\mu_{n}^{ \pm}=-\frac{\beta_{n}}{2} \pm \mathrm{i} \delta_{n}
$$


and we can write

$$
Q_{m n}=a\left(m-\mu_{n}^{+}\right)\left(m-\mu_{n}^{-}\right)
$$

Thus, using (3), we obtain

$$
\begin{aligned}
\mathcal{S} & =\frac{\pi}{a} \sum_{n} \frac{\mathrm{e}^{2 \pi \mathrm{i} n \eta}}{\mu_{n}^{-}-\mu_{n}^{+}}\left(\frac{\mathrm{e}^{\pi \mathrm{i}(2 \xi-1) \mu_{n}^{+}}}{\sin \pi \mu_{n}^{+}}-\frac{\mathrm{e}^{\pi \mathrm{i}(2 \xi-1) \mu_{n}^{-}}}{\sin \pi \mu_{n}^{-}}\right) \\
& =\frac{\pi}{a} \sum_{n} \frac{1}{\delta_{n}} \mathrm{e}^{-\pi \mathrm{i} \beta_{n} \xi+2 \pi \mathrm{i} n \eta} \frac{\sinh \left[2 \pi \delta_{n}(1-\xi)\right]+\mathrm{e}^{\pi \mathrm{i} \beta_{n}} \sinh 2 \pi \delta_{n} \xi}{\cosh 2 \pi \delta_{n}-\cos \beta_{n} \pi} .
\end{aligned}
$$

The apparent singularity if $\delta_{n}=0$, for a particular $n$, is removable (it corresponds to the case of a double pole) and so does not cause any difficulty. If $b=d=0$, then $\beta_{n}=0$ and

$$
\mathcal{S}=\frac{\pi}{a} \sum_{n} \frac{1}{\delta_{n}} \mathrm{e}^{2 \pi \mathrm{i} n \eta} \frac{\cosh \left[\pi \delta_{n}(1-2 \xi)\right]}{\sinh \pi \delta_{n}} .
$$

If we write $\Delta=\sqrt{a c-b^{2}}$, we see that

$$
\delta_{n} \sim \Delta|n| / a \quad \text { as }|n| \rightarrow \infty .
$$

Hence, provided $\xi \neq 0$, (17) expresses the original two-dimensional conditionally convergent sum as a single exponentially convergent sum. If we write

$$
\xi^{*}=\min (\xi, 1-\xi)=1 / 2-|1 / 2-\xi|,
$$

then the terms in the sum decay like $n^{-1} \exp \left(-2 \pi \xi^{*} \Delta|n| / a\right)$. Clearly, in order to achieve the best possible convergence, we should, therefore, determine which of the indices to treat as $m$ and which as $n$ to ensure that $|1 / 2-\xi| \leq|1 / 2-\eta|$.

A standard technique for avoiding the problems caused by the conditional convergence of $\mathcal{S}$ is to generalise the sum and instead consider

$$
\mathcal{S}_{s}=\sum_{m, n} Q_{m n}^{-s} \mathrm{e}^{2 \pi \mathrm{i}(m \xi+n \eta)},
$$

which converges absolutely for $\operatorname{Re} s>1$. The expression we have derived above should then correspond to setting $s=1$. Note that the results in Borwein et al. (1998) essentially tell us that this sum is well behaved, in the sense that the sum is independent of any reasonable ordering of the terms, provided $\operatorname{Re} s>1 / 2$.

We can evaluate $\mathcal{S}_{s}$ for $\operatorname{Re} s>1$ by modifying the method of Glasser (1974) to accommodate the indefinite nature of $Q_{m n}$. First, we split the sum into two depending on the sign of $\delta_{n}^{2}$,

$$
\mathcal{S}_{s}=\mathcal{S}_{s}^{-}+\mathcal{S}_{s}^{+}=\sum_{m} \sum_{\delta_{n}^{2}<0} Q_{m n}^{-s} \mathrm{e}^{2 \pi \mathrm{i}(m \xi+n \eta)}+\sum_{m} \sum_{\delta_{n}^{2}>0} Q_{m n}^{-s} \mathrm{e}^{2 \pi \mathrm{i}(m \xi+n \eta)} .
$$

For $\mathcal{S}_{s}^{+}$, we express the summand as a Mellin transform and interchange summation and integration,

$$
\mathcal{S}_{s}^{+}=\frac{1}{\Gamma(s)} \int_{0}^{\infty} x^{s-1} \sum_{m} \sum_{\delta_{n}^{2}>0} \mathrm{e}^{2 \pi \mathrm{i}(m \xi+n \eta)-Q_{m n} x} \mathrm{~d} x .
$$

Note that $a^{2} \delta_{n}^{2}=a Q_{m n}-(a m+b n+d / 2)^{2}$ and so $\delta_{n}^{2}>0$ implies $Q_{m n}>0$.

Jacobi's imaginary transformation in the form (Whittaker and Watson, 1927, p. 476)

$$
\sum_{m} \mathrm{e}^{-m^{2} x} \mathrm{e}^{2 m \mathrm{i} z}=\left(\frac{\pi}{x}\right)^{1 / 2} \sum_{m} \mathrm{e}^{-(m \pi+z)^{2} / x} \quad x>0
$$

shows that, for each $n$,

$$
\sum_{m} \mathrm{e}^{2 \pi \mathrm{i}(m \xi+n \eta)-Q_{m n} x}=\left(\frac{\pi}{a x}\right)^{1 / 2} \sum_{m} \mathrm{e}^{-\frac{\pi^{2}}{a x}(m+\xi)^{2}-\delta_{n}^{2} a x-\pi \mathrm{i} \beta_{n}(m+\xi)+2 \pi \mathrm{i} n \eta},
$$


valid for all $x \in(0, \infty)$. If we use this in $(23)$, we get

$$
\mathcal{S}_{S}^{+}=\frac{\pi^{1 / 2}}{a^{1 / 2} \Gamma(s)} \int_{0}^{\infty} x^{s-3 / 2} \sum_{m} \sum_{\delta_{n}^{2}>0} \mathrm{e}^{-\frac{\pi^{2}}{a x}(m+\xi)^{2}-\delta_{n}^{2} a x-\pi \mathrm{i} \beta_{n}(m+\xi)+2 \pi \mathrm{i} n \eta} \mathrm{d} x .
$$

Integration and summation can again be interchanged and the integral can be evaluated in terms of modified Bessel functions (Gradshteyn and Ryzhik, 2000, 3.486(4)), leading to

$$
\begin{aligned}
\mathcal{S}_{s}=\frac{2 \pi^{s}}{a^{s} \Gamma(s)} \sum_{m} \sum_{\delta_{n}^{2}>0}\left|\frac{m+\xi}{\delta_{n}}\right|^{s-1 / 2} \mathrm{~K}_{s-1 / 2}\left(2 \pi\left|\delta_{n}(m+\xi)\right|\right) \mathrm{e}^{-\pi \mathrm{i} \beta_{n}(m+\xi)+2 \pi \mathrm{i} n \eta} & \\
& +\sum_{m} \sum_{\delta_{n}^{2}<0} Q_{m n}^{-s} \mathrm{e}^{2 \pi \mathrm{i}(m \xi+n \eta)} .
\end{aligned}
$$

The first sum then converges exponentially in both $m$ and $n$. The second sum is finite in $n$, but still contains an infinite sum over $m$ which converges like $m^{-2 s} \mathrm{e}^{2 \pi \mathrm{i} m \xi}$. The first person to establish the relationship between phase-modulated sums (the sum over $m$ in (21)) and sums of modified Bessel functions appears to have been Kober (1935).

We now specialise to the case $s=1$ (and write $\mathcal{S}$ for $\mathcal{S}_{1}$ ). Note that $\mathrm{K}_{1 / 2}(z)=\mathrm{e}^{-z} \sqrt{\pi / 2 z}$. From (27), we have

$$
\mathcal{S}=\frac{\pi}{a} \sum_{m} \sum_{\delta_{n}^{2}>0} \frac{1}{\delta_{n}} \mathrm{e}^{-2 \pi \delta_{n}|m+\xi|} \mathrm{e}^{-\pi \mathrm{i} \beta_{n}(m+\xi)+2 \pi \mathrm{i} n \eta}+\sum_{m} \sum_{\delta_{n}^{2}<0} Q_{m n}^{-1} \mathrm{e}^{2 \pi \mathrm{i}(m \xi+n \eta)} .
$$

Since $m+\xi \geq 0$ for $m=0,1,2, \ldots$ and $m+\xi \leq 0$ for $m=-1,-2,-3, \ldots$, the first sum over $m$ can be split at $m=0$ into two geometric progressions that can be summed explicitly. The second sum, which is finite in $n$, is evaluated using (3). This leads to (17) as before.

Cases where $Q_{m n}$ vanishes can be handled by using the appropriate results from Sec. II. We will illustrate by means of a simple example. Consider

$$
\mathcal{S}=\sum_{m, n} \frac{\mathrm{e}^{2 \pi \mathrm{i}(m \xi+n \eta)}}{m^{2}+n^{2}-p},
$$

where $p$ is a positive integer and any terms where $m^{2}+n^{2}=p$ are omitted. For a particular $n$ there are three possibilities; either $n^{2}=p$, in which case we use (8) with $k=0$, or $n^{2}-p=-k^{2}$ for some non-zero integer $k$, in which case we use (7), or neither of these, in which case we use (18). Thus,

$$
\sum_{m, n}^{\prime} \frac{\mathrm{e}^{2 \pi \mathrm{i}(m \xi+n \eta)}}{m^{2}+n^{2}-p}=\sum_{n} \mathrm{e}^{2 \pi \mathrm{i} n \eta} f_{n}
$$

where

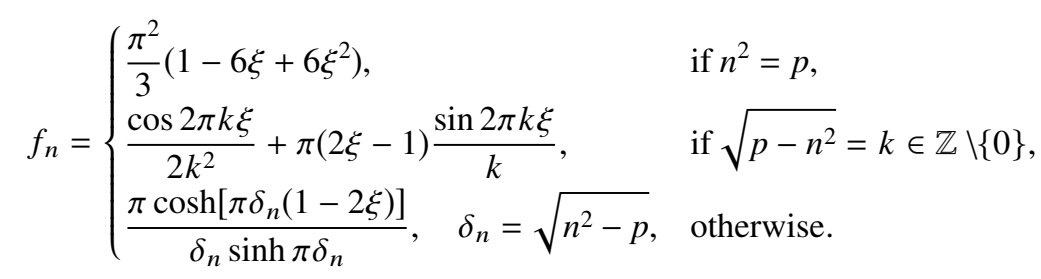

For sufficiently large $|n|$, the third condition always applies and so (30) gives an exponentially convergent representation for the sum.

\section{THE HELMHOLTZ GREEN'S FUNCTION FOR A TWO-DIMENSIONAL LATTICE}

A quasi-periodic Green's function is an array of sources modulated by a phase factor governed by a Bloch vector $\boldsymbol{\beta}$. For the Helmholtz equation with frequency parameter $\kappa$, this is a doubly 
infinite sum of phase modulated Hankel functions. Formally, the Green's function can be expressed as the spatial sum

$$
G(\mathbf{r})=-\frac{\mathrm{i}}{4} \sum_{\mathbf{R}_{m n} \in \Lambda} \mathrm{H}_{0}\left(\kappa\left|\mathbf{r}-\mathbf{R}_{m n}\right|\right) \mathrm{e}^{\mathrm{i} \boldsymbol{\beta} \cdot \mathbf{R}_{m n}} .
$$

Here, $\Lambda$ is a two-dimensional lattice, the points of which have position vectors

$$
\mathbf{R}_{m n}=m \mathbf{s}_{1}+n \mathbf{s}_{2}, \quad m \in \mathbb{Z}, n \in \mathbb{Z} .
$$

The vectors $\mathbf{s}_{1}$ and $\mathbf{s}_{2}$ are linearly independent but otherwise arbitrary. Since the difference between any two lattice vectors $\mathbf{R}_{m n}$ and $\mathbf{R}_{i j}$ is also a lattice vector, it follows from (32) that

$$
G\left(\mathbf{r}+\mathbf{R}_{i j}\right)=\mathrm{e}^{\mathrm{i} \boldsymbol{\beta} \cdot \mathbf{R}_{i j}} G(\mathbf{r}),
$$

which demonstrates the quasi-periodicity of $G$. This function occurs naturally in a number of physical contexts when considering the propagation of waves through a doubly periodic medium. An example of how the quasi-periodic Green's function can be used in a sophisticated numerical scheme can be found in Barnett and Greengard (2010).

Reciprocal lattice vectors are defined via

$$
\mathbf{K}_{m n}=2 \pi\left(m \mathbf{b}_{1}+n \mathbf{b}_{2}\right), \quad m \in \mathbb{Z}, n \in \mathbb{Z},
$$

where

$$
\mathbf{s}_{i} \cdot \mathbf{b}_{j}=\delta_{i j}, \quad i, j=1,2 .
$$

The crucial property of the reciprocal lattice vectors is that for any integers $i, j, m, n$,

$$
\mathrm{e}^{\mathrm{i} \mathbf{R}_{m n} \cdot \mathbf{K}_{i j}}=1 .
$$

The area of a unit cell of the lattice $\Lambda$ is $\mathcal{A}=\left|\mathbf{s}_{1} \times \mathbf{s}_{2}\right|$. It follows from (37) that we can restrict $\beta$ to a single cell of the reciprocal lattice (or any equivalent region, such as the Brillouin zone (Brillouin, 1953, Chap. 6)). In other words, we can assume that $\beta=2 \pi\left(\sigma \mathbf{b}_{1}+\tau \mathbf{b}_{2}\right)$ where $0 \leq \sigma<1$ and $0 \leq \tau<1$.

The Poisson summation formula can be used to express $G$ as a sum over the reciprocal lattice, $\Lambda^{*}$. Such series are called dual, or spectral, series. We choose an appropriate integral representation for the Hankel function $\mathrm{H}_{0}$, substitute this into (32), and use the Poisson summation formula (see, for example, Linton (2010, Sec. 2)). If we define

$$
\boldsymbol{\beta}_{m n}=\boldsymbol{\beta}+\mathbf{K}_{m n}, \quad \beta_{m n}=\left|\boldsymbol{\beta}_{m n}\right|,
$$

we obtain

$$
G=\frac{1}{\mathcal{A}} \sum_{\mathbf{K}_{m n} \in \Lambda^{*}} \frac{\mathrm{e}^{\mathrm{i} \mathbf{r} \cdot \boldsymbol{\beta}_{m n}}}{\kappa^{2}-\beta_{m n}^{2}}=-\frac{\mathrm{e}^{\mathrm{i} \mathbf{r} \cdot \boldsymbol{\beta}}}{\mathcal{A}} \sum_{\mathbf{K}_{m n} \in \Lambda^{*}} \frac{\mathrm{e}^{\mathrm{i} \mathbf{r} \cdot \mathbf{K}_{m n}}}{\beta_{m n}^{2}-\kappa^{2}} .
$$

For the purposes of calculation, let us fix $\mathbf{r}=\xi \mathbf{s}_{1}+\eta \mathbf{s}_{2}$, with $\xi$ and $\eta$ both in $[0,1)$ though not both zero, so that

$$
\mathbf{r} \cdot \mathbf{K}_{m n}=2 \pi\left(\xi \mathbf{s}_{1}+\eta \mathbf{s}_{2}\right) \cdot\left(m \mathbf{b}_{1}+n \mathbf{b}_{2}\right)=2 \pi(m \xi+n \eta)
$$

and we have, with $\beta=|\boldsymbol{\beta}|$,

$$
\beta_{m n}^{2}=\beta^{2}+4 \pi \boldsymbol{\beta} \cdot\left(m \mathbf{b}_{1}+n \mathbf{b}_{2}\right)+4 \pi^{2}\left(m \mathbf{b}_{1}+n \mathbf{b}_{2}\right)^{2} .
$$

Based on the discussion after Eq. (20), we will achieve the best possible convergence if we ensure that $|1 / 2-\xi| \leq|1 / 2-\eta|$ which we can always do by interchanging $\xi, \eta$ and $m, n$ if necessary. Then,

$$
\sum_{\mathbf{K}_{m n} \in \Lambda^{*}} \frac{\mathrm{e}^{\mathrm{i} \mathbf{r} \cdot \mathbf{K}_{m n}}}{\beta_{m n}^{2}-\kappa^{2}} \equiv \sum_{m, n} \frac{\mathrm{e}^{2 \pi \mathrm{i}(m \xi+n \eta)}}{a m^{2}+2 b m n+c n^{2}+d m+e n+f},
$$

where, with $b_{i}=\left|\mathbf{b}_{i}\right|, i=1,2$,

$a=4 \pi^{2} b_{1}^{2}, \quad b=4 \pi^{2} \mathbf{b}_{1} \cdot \mathbf{b}_{2}, \quad c=4 \pi^{2} b_{2}^{2}, \quad d=4 \pi \boldsymbol{\beta} \cdot \mathbf{b}_{1}, \quad e=4 \pi \boldsymbol{\beta} \cdot \mathbf{b}_{2}, \quad f=\beta^{2}-\kappa^{2}$. 
Since $\mathbf{b}_{1}$ and $\mathbf{b}_{2}$ cannot be parallel, $a c-b^{2}>0$ and we have

$$
\Delta=4 \pi^{2} \sqrt{b_{1}^{2} b_{2}^{2}-\left(\mathbf{b}_{1} \cdot \mathbf{b}_{2}\right)^{2}}=4 \pi^{2} b_{1} b_{2} \sin \chi,
$$

where $\chi \in(0, \pi)$ is the angle between the vectors $\mathbf{b}_{1}$ and $\mathbf{b}_{2}$. Simple calculations then show that

$$
a e-b d=2 \tau \Delta^{2}, \quad a f-d^{2} / 4=\tau^{2} \Delta^{2}-4 \pi^{2} b_{1}^{2} \kappa^{2},
$$

and hence, $\beta_{n}$ and $\delta_{n}^{2}$, defined in (12) and (13), are given by

$$
\begin{aligned}
& \beta_{n}=2\left(\sigma+\frac{n+\tau}{b_{1}^{2}} \mathbf{b}_{1} \cdot \mathbf{b}_{2}\right), \\
& \delta_{n}^{2}=\frac{1}{16 \pi^{4} b_{1}^{4}}\left(\Delta^{2}(n+\tau)^{2}-4 \pi^{2} b_{1}^{2} \kappa^{2}\right) .
\end{aligned}
$$

With these values for $\beta_{n}$ and $\delta_{n}^{2}$ (if one of $\xi$ or $\eta$ is zero, we can assume it is $\eta$ ), we then have the exponentially convergent sum

$$
G=-\frac{\pi \mathrm{e}^{\mathrm{i} r \cdot \beta}}{a \mathcal{A}} \sum_{n} \frac{1}{\delta_{n}} \mathrm{e}^{-\pi \mathrm{i} \beta_{n} \xi+2 \pi \mathrm{i} n \eta} \frac{\sinh \left[2 \pi \delta_{n}(1-\xi)\right]+\mathrm{e}^{\pi \mathrm{i} \beta_{n}} \sinh 2 \pi \delta_{n} \xi}{\cosh 2 \pi \delta_{n}-\cos \beta_{n} \pi} .
$$

For each value of $n$, there are singularities, whenever $\pm \mathrm{i} \delta_{n}-\beta_{n} / 2=m$ is an integer, equivalent to pairs $(m, n)$ for which $\kappa^{2}=\beta_{m n}^{2}$. These singularities correspond to resonances of the unit cell of the lattice (i.e., eigenvalues of the negative Laplacian on a cell of the lattice with quasi-periodic boundary conditions; see Ham and Segall (1961) and Barnett and Greengard (2010)). In the context of photonic crystals, they can be thought of as the result of the dispersion relation intersecting a light line. The terms in the sum decay like $n^{-1} \exp \left(-2 \pi \xi^{*}|n|\left(b_{2} / b_{1}\right) \sin \chi\right)$, and so, for a given lattice, convergence will deteriorate as the position vector $\mathbf{r}$ approaches points of the lattice (as this corresponds to $\xi^{*} \rightarrow 0$ ). These are points at which, from (32), $G$ has a logarithmic singularity.

\section{REPRESENTATIONS IN TERMS OF $\theta$-FUNCTIONS}

From (17), noting that $\delta_{n}$ is real and positive for sufficiently large $n$, we can expand the denominator in powers of $\mathrm{e}^{-2 \pi \delta_{n}}$ to yield

$$
\begin{aligned}
\mathcal{S}=\frac{\pi}{a} \sum_{n} \frac{1}{\delta_{n}} \mathrm{e}^{2 \pi \mathrm{i} n \eta} \mathrm{e}^{-\pi \mathrm{i} \beta_{n} \xi} \sum_{j=0}^{\infty}\left(\mathrm{e}^{-2 \pi \delta_{n}(j+\xi)}-\mathrm{e}^{-2 \pi \delta_{n}(2+j-\xi)}\right. & \\
& \left.+\mathrm{e}^{\pi \mathrm{i} \beta_{n}}\left(\mathrm{e}^{-2 \pi \delta_{n}(j+1-\xi)}-\mathrm{e}^{-2 \pi \delta_{n}(j+1+\xi)}\right)\right) \mathrm{U}_{j}\left(\cos \beta_{n} \pi\right),
\end{aligned}
$$

where $\mathrm{U}_{j}(\cos \theta)=\sin [(j+1) \theta] / \sin \theta$ is a Chebyshev polynomial of the second kind. This can be rearranged, taking advantage of properties of the Chebyshev polynomials: $\mathrm{U}_{0}(x)=1, \mathrm{U}_{1}(x)=2 x$, $\mathrm{U}_{j}(x)-x \mathrm{U}_{j-1}(x)=x \mathrm{U}_{j-1}(x)-\mathrm{U}_{j-2}(x)=\mathrm{T}_{j}(x), \mathrm{T}_{j}(\cos \theta)=\cos j \theta$, to yield

$$
\mathcal{S}=\frac{\pi}{a} \sum_{n} \frac{1}{\delta_{n}} \mathrm{e}^{2 \pi \mathrm{i} n \eta} \mathrm{e}^{-\pi \mathrm{i} \beta_{n} \xi}\left(\mathrm{e}^{-2 \pi \xi \delta}+\sum_{j=1}^{\infty}\left(\mathrm{e}^{-\pi \mathrm{i} j \beta_{n}} \mathrm{e}^{-2 \pi(j+\xi) \delta_{n}}+\mathrm{e}^{\pi \mathrm{i} j \beta_{n}} \mathrm{e}^{-2 \pi(j-\xi) \delta_{n}}\right)\right) .
$$

This representation is exponentially convergent in both $j$ and $n$ and holds for any sum of form (9) with $Q_{m n}$ as in (10).

If $\delta_{n}^{2}$ is a multiple of $(n+v)^{2}$ for some rational number $v$, then (50) can be evaluated in terms of $\theta$-functions. Since $Q_{m n}=a\left(\left(m+\beta_{n} / 2\right)^{2}+\delta_{n}^{2}\right)$, we consider sums of the form

$$
\mathcal{S}=\sum_{m, n} \frac{\mathrm{e}^{2 \pi \mathrm{i}(m \xi+n \eta)}}{x^{2}(m+\mu+\lambda(n+v))^{2}+(n+v)^{2}}
$$


for which $\beta_{n}=2(\mu+\lambda n+\lambda v)$ and $\delta_{n}=|n+v| / x(x>0)$. Sums of this type form only a subset of those where $Q_{m n}$ is of form (10), in particular $Q_{m n}$ must be positive definite, but the results derived in this section would appear to extend significantly the available $\theta$-function representations for phase-modulated lattice sums.

Below, we consider three separate cases. First, we consider the case when $v \in \mathbb{Q}$ is not an integer. In this case, the denominator never vanishes, whatever the values of $\lambda$ and $\mu$ and they can take arbitrary real values. However, in order to derive a $\theta$-function representation, we need to insist that $\mu$ is rational (it may take integer values). The second case is when $v$ is an integer. Clearly by shifting the index $n$, it is easy to express such a sum in terms of the $v=0$ case. We then need to insist that $\mu$ is not an integer to avoid a vanishing denominator, and in order to derive a $\theta$-function representation, we again need to consider rational values of $\mu$. Finally, we consider the case when $\mu=v=0$ with the $m=n=0$ term omitted. This latter case is equivalent to that considered in Stremler (2004) and will only be touched on briefly. Sums with other integer pairs $\mu$, $v$ (with the appropriate term omitted) are easily expressed in terms of this.

The numbering that we use for $\theta$-functions follows Whittaker and Watson (1927). A number of basic properties of $\theta_{i}(z, q), i=1,2,3,4$ will be used below and we collect them here for reference. Thus, if $\operatorname{Im} \tau>0$ and $q=\mathrm{e}^{\mathrm{i} \pi \tau}$, then

$$
\begin{gathered}
\theta_{1}(z+\pi, q)=-\theta_{1}(z, q), \quad \theta_{1}(z+\pi \tau, q)=-\mathrm{e}^{-2 \mathrm{i} z} \mathrm{e}^{-\pi \mathrm{i} \tau} \theta_{1}(z, q), \\
\theta_{1}(z+\pi / 2, q)=\theta_{2}(z, q), \quad \theta_{4}(z+\pi \tau / 2, q)=\mathrm{ie}^{-\mathrm{i} z} \mathrm{e}^{-\mathrm{i} \pi \tau / 4} \theta_{1}(z, q), \\
\theta_{3}(z+\pi \tau / 2, q)=\mathrm{e}^{-\mathrm{i} z} \mathrm{e}^{-\mathrm{i} \pi \tau / 4} \theta_{2}(z, q) .
\end{gathered}
$$

Taken together these identities allow us to express $\theta_{1}(z+m \pi / 2+n \pi \tau / 2, q)$ for any integers $m$ and $n$ as a multiple of one of the functions $\theta_{i}(z, q)$.

Sometimes, a particular sum can be evaluated in terms of $\theta$-functions in more than one way, leading to an identity between these functions, which is invariably equivalent to Jacobi's imaginary transformation. This transformation has already been used in series form in Sec. III. In terms of $\theta$-functions, this transformation gives rise to, for example,

$$
\begin{gathered}
\theta_{1}(z, q)=-\mathrm{i}(-\mathrm{i} \tau)^{-1 / 2} \mathrm{e}^{z^{2} / \pi \mathrm{i} \tau} \theta_{1}\left(\tau^{\prime} z, q^{\prime}\right), \quad|\arg (-\mathrm{i} \tau)|<\pi / 2, \\
\frac{\theta_{1}(z, q)}{\theta_{2}(z, q)}=-\mathrm{i} \frac{\theta_{1}\left(\tau^{\prime} z, q^{\prime}\right)}{\theta_{4}\left(\tau^{\prime} z, q^{\prime}\right)}, \quad \frac{\theta_{3}(z, q)}{\theta_{4}(z, q)}=\frac{\theta_{3}\left(\tau^{\prime} z, q^{\prime}\right)}{\theta_{2}\left(\tau^{\prime} z, q^{\prime}\right)},
\end{gathered}
$$

where $\tau^{\prime}=-1 / \tau$ and $q^{\prime}=\mathrm{e}^{\mathrm{i} \pi \tau^{\prime}}$.

\section{A. Case 1: $v=q / p$, where $q$ and $p$ are integers and $p$ does not divide $q$}

Define $\zeta_{p}=\mathrm{e}^{2 \pi \mathrm{i} / p}$ (since $p$ does not divide $q$ we have $p>1$ ). We make use of the identity, valid for $|r|<1$,

$$
\sum_{n} \frac{\mathrm{e}^{2 \pi \mathrm{i} n \eta}}{|p n+q|} r^{|p n+q|}=-\frac{1}{p} \sum_{\ell=0}^{p-1} \zeta_{p}^{-q(\ell+\eta)} \ln \left[\left(1-\zeta_{p}^{\ell+\eta} r\right)\left(1-\zeta_{p}^{-\ell-\eta} r\right)\right] .
$$

One way to establish this identity is to expand the right-hand side as a Taylor series in $r$. One finds that the coefficient of $r^{m}$ is

$$
\frac{1}{p m}\left(\zeta_{p}^{\eta(m-q)} \sum_{\ell=0}^{p-1} \zeta_{p}^{\ell(m-q)}+\zeta_{p}^{-\eta(m+q)} \sum_{\ell=0}^{p-1} \zeta_{p}^{-\ell(m+q)}\right) .
$$

The first sum over $\ell$ is zero unless $(m-q) / p$ is an integer. If it is, there is an integer $n$ such that $m=p n+q$ and the sum equals $p$. Similarly, the second sum is zero unless there is an integer $n$ such that $p n+q=-m$ and then the sum is again $p$. 
If we use this in (50) with $\beta_{n}=2(\mu+\lambda n+\lambda v)$ and $\delta_{n}=|n+v| / x$, we obtain

$$
\begin{aligned}
\mathcal{S}=-\frac{\pi}{x} \mathrm{e}^{-2 \pi \mathrm{i} \mu \xi} \sum_{\ell=0}^{p-1} \zeta_{p}^{-q(\ell+\eta)}[ & \ln \left|1-\mathrm{e}^{2 \pi \mathrm{i} \alpha_{\ell}}\right|^{2} \\
& \left.+\sum_{j=1}^{\infty}\left(\mathrm{e}^{-2 \pi \mathrm{i} j \mu} \ln \left|1-\mathrm{e}^{2 \pi \mathrm{i} \alpha} \rho^{2 j}\right|^{2}+\mathrm{e}^{2 \pi \mathrm{i} j \mu} \ln \left|1-\mathrm{e}^{-2 \pi \mathrm{i} \alpha_{\ell}} \rho^{2 j}\right|^{2}\right)\right],
\end{aligned}
$$

where

$$
\alpha_{\ell}=\frac{1}{p}(\ell+\eta+\xi(\mathrm{i} / x-\lambda)), \quad \rho=\mathrm{e}^{\pi \mathrm{i}(\mathrm{i} / x-\lambda) / p} .
$$

Equation (56) is valid for all $\mu$, but in order to make further progress, we assume that $\mu=r / s, r$ and $s$ integers with $s>0$. The sum over $j$ can be split into $s$ separate sums depending on the value of $j \bmod s$ and each of these new sums can be written as a sum over all positive integers $k$ by suitably defining $k$ in each case. If we define

$$
T_{t}^{ \pm}=\sum_{k=1}^{\infty} \zeta_{s}^{ \pm r t} \ln \left|1-\mathrm{e}^{\mp 2 \pi \mathrm{i} \alpha_{\ell}} \rho^{2[s(k-1)+t]}\right|,
$$

then

$$
\mathcal{S}=-\frac{2 \pi}{x} \zeta_{s}^{-r \xi} \sum_{\ell=0}^{p-1} \zeta_{p}^{-q(\ell+\eta)}\left[\ln \left|\sin \alpha_{\ell}\right|+\sum_{t=1}^{s}\left(T_{t}^{-}+T_{t}^{+}\right)\right],
$$

where we have made use of the fact that $\sum_{\ell=0}^{p-1} \zeta_{p}^{-\ell q}=0$.

Now,

$$
T_{s}^{-}+T_{s}^{+}=\ln \prod_{k=1}^{\infty}\left|1-2 \rho^{2 s k} \cos 2 \pi \alpha_{\ell}+\rho^{4 s k}\right|=\ln \left|\frac{\theta_{1}\left(\pi \alpha_{\ell}, \rho^{s}\right)}{2 G \rho^{s / 4} \sin \pi \alpha_{\ell}}\right|,
$$

where $G$ is defined via $2 \rho^{s / 4} G^{3}=\theta_{1}^{\prime}\left(0, \rho^{s}\right) \quad$ (Whittaker and Watson, 1927, Secs. 21.3 and 21.42). The factor $2 G \rho^{s / 4}$ can, however, be ignored because it vanishes when the sum over $\ell$ is performed. The rest of the sum over $t$ can be evaluated by noting that

$$
\begin{aligned}
\sum_{t=1}^{s-1}\left(T_{t}^{-}+T_{t}^{+}\right) & =\sum_{t=1}^{s-1}\left(T_{s-t}^{-}+T_{t}^{+}\right) \\
& =\sum_{t=1}^{s-1} \zeta_{s}^{r t} \ln \prod_{k=1}^{\infty}\left|1-2 \rho^{s(2 k-1)} \cos \left[2 \pi\left(\alpha_{\ell}+\tau(1 / 2-t / s)\right)\right]+\rho^{2 s(2 k-1)}\right| \\
& =\sum_{t=1}^{s-1} \zeta_{s}^{r t}\left(\ln \left|\theta_{4}\left(\pi\left(\alpha_{\ell}+\tau(1 / 2-t / s)\right), \rho^{s}\right)\right|-\ln |G|\right),
\end{aligned}
$$

where $\tau=s(\mathrm{i} / x-\lambda) / p$ (so that $\rho^{s}=\mathrm{e}^{\pi \mathrm{i} \tau}$ ). The function $\theta_{4}$ can be expressed in terms of $\theta_{1}$ using (52) and we note that the modulus of the resulting factor multiplying $\theta_{1}$ does not depend on $\ell$ and so vanishes when the sum over $\ell$ is performed. Putting everything together yields the final result

$$
\sum_{m, n} \frac{\mathrm{e}^{2 \pi \mathrm{i}[(m+\mu) \xi+(n+v) \eta]}}{x^{2}(m+\mu+\lambda(n+v))^{2}+(n+v)^{2}}=-\frac{2 \pi}{x} \sum_{\ell=0}^{p-1} \sum_{t=0}^{s-1} \mathrm{e}^{2 \pi \mathrm{i}(t \mu-\ell v)} \ln \left|\theta_{1}\left(\pi \alpha_{\ell t}, \rho^{s}\right)\right|,
$$

where $\mu=r / s, v=q / p$ and

$$
\alpha_{\ell t}=\frac{1}{p}(\ell+\eta+(t-\xi)(\lambda-\mathrm{i} / x)), \quad \rho=\mathrm{e}^{\pi \mathrm{i}(\mathrm{i} / x-\lambda) / p} .
$$

Alternatively,

$$
\pi \alpha_{\ell t}=z+\frac{\pi \ell}{p}-\frac{\pi t \tau}{s}, \quad \rho^{s}=\mathrm{e}^{\pi \mathrm{i} \tau}, \quad z=\frac{\pi \eta}{p}+\frac{\pi \xi \tau}{s} .
$$


In this form, we can see that our original sum has been expressed as a sum involving the evaluation of a $\theta$-function at $p s$ points distributed across the fundamental parallelogram of the $\theta$-function shifted by the complex number $z$.

The singularity as $(\xi, \eta) \rightarrow(0,0)$ is easily extracted since $\theta_{1}(w, \rho) \sim \theta_{1}^{\prime}(0, \rho) w+O\left(w^{2}\right)$ as $w \rightarrow 0$. The zeros of $\theta_{1}\left(w, \mathrm{e}^{\pi \mathrm{i} \tau}\right)$ occur at $w=(m+n \tau) \pi$ for integers $m$ and $n$. Hence, the leadingorder behaviour in $(64)$ as $(\xi, \eta) \rightarrow(0,0)$ comes from the $\ell=t=0$ term and we get the logarithmic singularity $-(\pi / x) \ln \left[(\eta-\lambda \xi)^{2}+\xi^{2} / x^{2}\right]$. Note that (64) is valid if $r=0$, in which case the right-hand side is independent of the value chosen for $s$, though this is far from obvious.

Equation (64) is a generalisation of results that appear in Berndt et al. (2012) for the case $\lambda=0$, $\mu \equiv r / s=0$, and $\xi=\eta=1 / 2$. In the case where $q / p=r / s=1 / 2$, the sum can be cast as a sum over odd integers, and for $\lambda=0$, this case was considered recently in Bailey and Borwein (2013). From (64), we can show

$$
\sum_{m, n \text { odd }} \frac{\mathrm{e}^{\pi \mathrm{i}(m \xi+n \eta)}}{x^{2}(m+\lambda n)^{2}+n^{2}}=\frac{\pi}{2 x} \ln \left|\frac{\theta_{2}\left(z, \mathrm{e}^{-\pi / x-\pi \mathrm{i} \lambda}\right) \theta_{4}\left(z, \mathrm{e}^{-\pi / x-\pi \mathrm{i} \lambda}\right)}{\theta_{1}\left(z, \mathrm{e}^{-\pi / x-\pi \mathrm{i} \lambda}\right) \theta_{3}\left(z, \mathrm{e}^{-\pi / x-\pi \mathrm{i} \lambda}\right)}\right|,
$$

where $z=\pi(\eta-\lambda \xi+\mathrm{i} \xi / x) / 2$, in agreement with Bailey and Borwein (2013, Eq. (47)) when $\lambda=0$. For specific values of the parameters, it is sometimes possible to express the combination of $\theta$-functions that appear in formulas of this type as the root of a polynomial equation and hence evaluate the sum as the logarithm of an algebraic number. A particularly nice example, which we reproduce here from Bailey and Borwein (2013), is given by setting $x^{2}=3, \xi=\lambda=0$, and $\eta=1 / 3$ in (67), whence it can be shown that

$$
\sum_{m, n \text { odd }} \frac{\mathrm{e}^{n \pi \mathrm{i} / 3}}{3 m^{2}+n^{2}}=\frac{\pi \ln 3}{8 \sqrt{3}}
$$

When $\lambda=0$ and $x=1$, sums of form (51) can be related to the displaced lattice sums investigated by McPhedran et al. (2007); see also Chaps. 3 and 4 of Borwein et al. (2013). The most significant difference between their work and that presented here is that our focus is on phasemodulated sums rather than raising the denominator to the power $s$ (not to be confused with the integer $s$ in the rest of this section) and relating the sums to Dirichlet $L$-series. As the most simple example, if we set $\xi=\eta=0$ (but raise the denominator to the power $s$ so that we have convergence provided $\operatorname{Re} s>1$ ), then Glasser (1973) showed that $\sum_{m, n \text { odd }}\left(m^{2}+n^{2}\right)^{-s}=2^{2-s}\left(1-2^{-s}\right) \zeta(s) \beta(s)$, where $\zeta(s)$ is the Riemann zeta function and $\beta(s)$ is Dirichlet's beta function defined for $\operatorname{Re} s>0$ by $\sum_{n=0}^{\infty}(-1)^{n} /(2 n+1)^{s}$. This shows the singular nature of the sum as $s \rightarrow 1+$ since $\zeta(s)$ has a simple pole at $s=1$ whilst $\beta(1)=\pi / 4$.

Returning to (64), cases where either or both of $p$ and $s$ are greater than 2 lead to more complicated expressions. As an illustration, we provide two examples,

$$
\begin{aligned}
\sum_{m, n} \frac{\mathrm{e}^{2 \pi \mathrm{i}[(m+1 / 2) \xi+(n+1 / 4) \eta]}}{x^{2}(m+1 / 2)^{2}+(n+1 / 4)^{2}}=\frac{2 \pi}{x} \ln \left|\frac{\theta_{2}\left(z, \mathrm{e}^{-\pi / 2 x}\right) \theta_{4}\left(z, \mathrm{e}^{-\pi / 2 x}\right)}{\theta_{1}\left(z, \mathrm{e}^{-\pi / 2 x}\right) \theta_{3}\left(z, \mathrm{e}^{-\pi / 2 x}\right)}\right| \\
+\frac{2 \pi \mathrm{i}}{x} \ln \left|\frac{\theta_{1}\left(\tilde{z}, \mathrm{e}^{-\pi / 2 x}\right) \theta_{3}\left(\tilde{z}, \mathrm{e}^{-\pi / 2 x}\right)}{\theta_{2}\left(\tilde{z}, \mathrm{e}^{-\pi / 2 x}\right) \theta_{4}\left(\tilde{z}, \mathrm{e}^{-\pi / 2 x}\right)}\right|,
\end{aligned}
$$

where $z=(\pi / 4)(\eta+\mathrm{i} \xi / x)$ and $\tilde{z}=z+\pi / 4$, and

$$
\begin{aligned}
\sum_{m, n} \frac{\mathrm{e}^{2 \pi \mathrm{i}[(m+1 / 3) \xi+(n+1 / 3) \eta]}}{x^{2}(m+1 / 3)^{2}+(n+1 / 3)^{2}}=- & \frac{2 \pi}{x} \ln \left|\Theta_{00} \Theta_{11} \Theta_{22}\right| \\
& +\frac{\pi}{x} \ln \left|\Theta_{01} \Theta_{10} \Theta_{12} \Theta_{21} \Theta_{02} \Theta_{20}\right|-\frac{\pi \mathrm{i} \sqrt{3}}{x} \ln \left|\frac{\Theta_{01} \Theta_{12} \Theta_{20}}{\Theta_{10} \Theta_{21} \Theta_{02}}\right|,
\end{aligned}
$$

where we have written

$$
\Theta_{m, n}=\theta_{1}\left(\frac{\pi}{3}(\eta+\mathrm{i} \xi / x+m-\mathrm{i} n / x), \mathrm{e}^{-\pi / x}\right)
$$


The techniques used in Bailey and Borwein (2013) can no doubt be extended to allow expressions like those that appear on the right-hand sides of (69) and (70) to be written in terms of logarithms of algebraic numbers for specific rational values of $\xi, \eta$, and $x$.

\section{B. Case 2: $v=0, \mu$ not an integer}

In this case, $\beta_{n}=2(\mu+\lambda n)$ and $\delta_{n}=|n| / x$. The $n=0$ term in (17) needs to be treated separately, using (4), and then the other terms are expanded as in (50). We can then make use of the series expansion $\ln (1-z)=-\sum_{n=1}^{\infty} z^{n} / n$, valid provided $|z| \leq 1$ and $z \neq 1$. In this way, we can show that

$$
\begin{aligned}
\mathcal{S}=\frac{\pi^{2} \mathrm{e}^{-2 \pi \mathrm{i} \mu \xi}}{x^{2} \sin ^{2} \pi \mu}\left(1-\xi+\xi \mathrm{e}^{2 \pi \mathrm{i} \mu}\right) & -\frac{2 \pi}{x} \mathrm{e}^{-2 \pi \mathrm{i} \mu \xi}\left[\ln \left|1-\mathrm{e}^{2 \pi \mathrm{i} \alpha}\right|\right. \\
& \left.+\sum_{j=1}^{\infty}\left(\mathrm{e}^{-2 \pi \mathrm{i} j \mu} \ln \left|1-\mathrm{e}^{2 \pi \mathrm{i} \alpha} \rho^{2 j}\right|+\mathrm{e}^{2 \pi \mathrm{i} j \mu} \ln \left|1-\mathrm{e}^{-2 \pi \mathrm{i} \alpha} \rho^{2 j}\right|\right)\right],
\end{aligned}
$$

where $\alpha=\eta-\lambda \xi+\mathrm{i} \xi / x$ and $\rho=\mathrm{e}^{-\pi / x} \mathrm{e}^{-\pi \mathrm{i} \lambda}$

Once again, we assume that $\mu=r / s, r$ and $s$ integers, though now we need to insist that $\mu$ is not an integer. The analysis is essentially the same as before but now there is no sum over $\ell$ to knock out the factors which appear when the infinite products are written in terms of $\theta$-functions. However, $\sum_{t=0}^{s-1} \zeta_{s}^{r t}=0$ and thus we find that

$$
\begin{aligned}
\mathcal{S}=\frac{\pi^{2} \mathrm{e}^{-2 \pi \mathrm{i} \mu \xi}}{x^{2} \sin ^{2} \pi \mu}\left(1-\xi+\xi \mathrm{e}^{2 \pi \mathrm{i} \mu}\right)-\frac{2 \pi}{x} \mathrm{e}^{-2 \pi \mathrm{i} \mu \xi}[ & \frac{\pi}{x}\left(\frac{s}{4}-\xi\right)+\ln \left|\theta_{1}\left(\pi \alpha, \rho^{s}\right)\right| \\
& \left.+\sum_{t=1}^{s-1} \zeta_{s}^{r t}\left(\ln \left|\theta_{4}\left(\pi(\alpha+\tau(1 / 2-t / s)), \rho^{s}\right)\right|\right)\right] .
\end{aligned}
$$

If we now express $\theta_{4}$ in terms of $\theta_{1}$ and note that

$$
\sum_{t=1}^{s-1} t \zeta_{s}^{r t}=\frac{s}{\zeta_{s}^{r}-1},
$$

( $\mu=r / s$ is not an integer), we arrive at

$$
\begin{aligned}
\sum_{m, n} \frac{\mathrm{e}^{2 \pi \mathrm{i}[(m+\mu) \xi+n \eta]}}{x^{2}(m+\mu+\lambda n)^{2}+n^{2}}=\frac{\pi^{2}}{x^{2} \sin ^{2} \pi \mu}\left[1+\left(\xi-\frac{s}{2}\right)\left(1-\mathrm{e}^{-2 \pi \mathrm{i} \mu}\right)\right] \\
-\frac{2 \pi}{x} \sum_{t=0}^{s-1} \mathrm{e}^{2 \pi \mathrm{i} t \mu} \ln \left|\theta_{1}\left(\pi \alpha-\pi t \tau / s, \mathrm{e}^{\pi \mathrm{i} \tau}\right)\right|,
\end{aligned}
$$

where $\tau=s(\mathrm{i} / x-\lambda)$.

If $\lambda=0$, we get nothing new here because the sum could equally well be evaluated from (64), the equivalence of the two resulting expressions being a consequence of (53). If we set $\mu \equiv r / s=1 / 2$ in (75), we obtain

$$
\begin{aligned}
\sum_{m, n} \frac{\mathrm{e}^{2 \pi \mathrm{i}[(m+1 / 2) \xi+n \eta]}}{x^{2}(m+\lambda n+1 / 2)^{2}+n^{2}} & =\frac{\pi^{2}}{x^{2}}(2 \xi-1)-\frac{2 \pi}{x} \ln \left|\frac{\theta_{1}\left(\pi \alpha, \mathrm{e}^{\pi \mathrm{i} \tau}\right)}{\theta_{1}\left(\pi \alpha-\pi \tau / 2, \mathrm{e}^{\pi \mathrm{i} \tau}\right)}\right| \\
& =-\frac{2 \pi}{x} \ln \left|\frac{\theta_{1}\left(\pi \alpha, \mathrm{e}^{-2 \pi / x-2 \pi \mathrm{i} \lambda}\right)}{\theta_{4}\left(\pi \alpha, \mathrm{e}^{-2 \pi / x-2 \pi \mathrm{i} \lambda}\right)}\right|,
\end{aligned}
$$

where we have used (52). 


\section{Case 3: $\mu=v=0$}

We consider

$$
\mathcal{S}=\sum_{m, n}^{\prime} \frac{\mathrm{e}^{2 \pi \mathrm{i}(m \xi+n \eta)}}{x^{2}(m+\lambda n)^{2}+n^{2}},
$$

where the $m=n=0$ term is omitted. Note that any sum with denominator $a m^{2}+2 b m n+c n^{2}$ and $a c>b^{2}$ can be written as a multiple of a sum of this type. We have $\beta_{n}=2 \lambda n, \delta_{n}=|n| / x$, and the only value of $n$ for which $-\beta_{n} / 2 \pm \mathrm{i} \delta_{n}$ is an integer is $n=0$. The sum over $m$ when $n=0$ is evaluated using (8) and we obtain

$\mathcal{S}=\frac{\pi^{2}}{3 x^{2}}\left(1-6 \xi+6 \xi^{2}\right)+\frac{\pi}{x} \sum_{n}^{\prime} \frac{1}{n} \mathrm{e}^{-2 \pi \mathrm{i} \lambda n \xi+2 \pi \mathrm{i} n \eta} \frac{\sinh [2 \pi n(1-\xi) / x]+\mathrm{e}^{2 \pi \mathrm{i} \lambda n} \sinh 2 \pi n \xi / x}{\cosh 2 \pi n / x-\cos 2 \lambda n \pi}$.

The sum can be treated as in the previous case except that now $\mu=0$ and so

$$
\mathcal{S}=\frac{\pi^{2}}{3 x^{2}}\left(1-6 \xi+6 \xi^{2}\right)-\frac{2 \pi}{x}\left[\ln \left|1-\mathrm{e}^{2 \pi \mathrm{i} \alpha}\right|+\ln \prod_{j=1}^{\infty}\left|1-2 \rho^{2 j} \cos 2 \pi \alpha+\rho^{4 j}\right|\right],
$$

where again $\alpha=\eta-\lambda \xi+\mathrm{i} \xi / x$ and $\rho=\mathrm{e}^{-\pi / x} \mathrm{e}^{-\pi \mathrm{i} \lambda}$. If we now write the infinite product as a $\theta$-function and simplify, we obtain the result

$$
\sum_{m, n}^{\prime} \frac{\mathrm{e}^{2 \pi \mathrm{i}(m \xi+n \eta)}}{x^{2}(m+\lambda n)^{2}+n^{2}}=\frac{2 \pi^{2} \xi^{2}}{x^{2}}-\frac{2 \pi}{3 x}\left(\ln 2+3 \ln \left|\theta_{1}(\pi \alpha, \rho)\right|-\ln \left|\theta_{1}^{\prime}(0, \rho)\right|\right),
$$

which is equivalent to Stremler (2004, Eq. (24)) and, with $\lambda=0$, to Borwein et al. (2013, Eq. (3.3.18)).

Bailey, D. H. and Borwein, J. M., Boundary Value Probl. 2013, 1.

Barnett, A. and Greengard, L., J. Comput. Phys. 229, 6898 (2010).

Berndt, B. C., Lamb, G., and Rogers, M., Ramanujan J. 29, 185 (2012).

Borwein, D., Borwein, J. M., and Pinner, C., Trans. Am. Math. Soc. 350, 3131 (1998).

Borwein, J. M., Glasser, M. L., McPhedran, R. C., Wan, J. G., and Zucker, I. J., Lattice Sums Then and Now, Encyclopedia of

Mathematics and its Applications (Cambridge University Press, 2013), Vol. 150.

Brillouin, L., Wave Propagation in Periodic Structures, 2nd ed. (Dover, 1953).

Dienstfrey, A., Hang, F., and Huang, J., Proc. R. Soc. London, Ser. A 457, 67 (2001).

Glasser, M. L., J. Math. Phys. 14, 409 (1973).

Glasser, M. L., J. Math. Phys. 15, 188 (1974).

Gradshteyn, I. S. and Ryzhik, I. M., Tables of Integrals, Series and Products, 6th ed. (Academic Press, New York, 2000).

Ham, F. S. and Segall, B., Phys. Rev. 124, 1786 (1961).

Jordan, K. E., Richter, G. R., and Sheng, P., J. Comput. Phys. 63, 222 (1986).

Kober, H., Math. Z. 39, 609 (1935).

Linton, C. M., SIAM Rev. 52, 630 (2010).

McPhedran, R. C., Botten, L. C., Nicorovici, N. A., and Zucker, I. J., J. Math. Phys. 48, 033501 (2007).

Moroz, A., J. Phys. A 39, 11247 (2006).

Stremler, M. A., J. Math. Phys. 45, 3584 (2004)

Whittaker, E. T. and Watson, G. N., A Course of Modern Analysis, 4th ed. (Cambridge University Press, 1927). 